\title{
ON MAXIMAL NONDETERMINING SUBALGEBRAS OF GROUP ALGEBRAS ${ }^{1}$
}

\section{SHU-SHIH BUTT}

Introduction. Let $L^{1}(G)$ be the group algebra of an arbitrary locally compact abelian group $G$. It is a regular semisimple self-adjoint commutative Banach algebra with the $L^{1}$-norm. The space of regular maximal ideals of $L^{1}(G)$ can be identified with the dual group (character group) $\Gamma=\hat{G}$ of $G$. The Gelfand transform of a function $f$ in $L^{1}(G)$ coincides then with the Fourier transform $\hat{f}$ of $f$.

A subalgebra $A$ of $L^{1}(G)$ is called nondetermining if $\hat{A}$ is not uniformly dense in $C_{0}(\Gamma)$ (cf. [2]). It is called irreducible if it does not contain any closed ideal of $L^{1}(G)$ with hull contained in proper closed subgroup of $\Gamma$. In case $A$ does not contain any ideal of $L^{1}(G)$ at all, it is called completely irreducible.

In this paper, we will first show that there is a reduction of maximal nondetermining subalgebras to irreducible ones. The reduced algebra will be a closed subalgebra of $L^{1}\left(G^{\prime}\right)$ where $G^{\prime}$ is a quotient group of $G$. Next we proved that for certain maximal nondetermining subalgebra $A$, we have $S(A)=\Gamma \subseteq M(A)$. Finally, we exhibit a maximal subalgebra of $L^{1}\left(Z^{\infty}\right)$ which is nondetermining and which does not belong to the two categories of maximal subalgebras previously known for discrete locally compact abelian groups, namely, a maximal subalgebra of $L^{1}\left(Z^{\infty}\right)$ which is neither associated with an order of $G$ nor gotten from an essential maximal subalgebra of $C(E)$ for a suitable Cantor set $E$ in $\Gamma$.

Throughout this paper, we adopt the terminology and notations of Rudin [4] and Hoffman \& Singer [3].

1. The reduction theorem. Before we establish the reduction of maximal nondetermining subalgebras to irreducible ones, let us recall that for every closed subgroup $\Lambda$ of $\Gamma$, there corresponds a closed subgroup $H$ of $G: H=\{x \in G:(x, \gamma)=1$ for all $\gamma \in \Lambda\}$. $H$ is called the annihilator of $\Lambda$ and is frequently denoted as $\Lambda^{\perp}$. It is shown that $\Lambda$ can be identified (topologically and algebraically) as the character group of the quotient group $G / H$. Dually, $\Lambda$ is called the annililator of $H$, and $H=(\Gamma / \Lambda)^{\perp}$.

Received by the editors July 21, 1967 and, in revised form, April 26, 1968.

1 This paper is a portion of the author's doctoral dissertation prepared at Tulane University under the direction of Dr. Frank Birtel. The author wishes to extend her sincere gratitude to him for his guidance and advice. 
It is also shown that when $\Lambda$ is a closed subgroup of $\Gamma$ and $H=\Lambda^{\perp}$, then $L^{1^{\wedge}}(G / H)=L^{1^{\wedge}}(G) \mid \Lambda[4$, Chapter 2]. So the inverse map $\pi$ of Fourier transform of $L^{1}(G / H)$ is well defined on $L^{1^{\wedge}}(G) \mid \Lambda$.

We may now state our main theorem.

THEOREM 1.1. If $A$ is a maximal nondetermining subalgebra of $L^{1}(G)$, then there exists a unique smallest closed subgroup $\Lambda$ of $\Gamma$ such that $\pi(\hat{A} \mid \Lambda)$ is an irreducible maximal nondetermining subalgebra of $L^{1}\left(G / \Lambda^{\perp}\right)$. Conversely, let $A^{\prime}$ be a maximal nondetermining subalgebra of $L^{1}\left(G^{\prime}\right)$ where $G^{\prime}=G / H$ and $H$ is a closed subgroup of $G$. Then the algebra $A=\left\{f \in L^{1}(G): \hat{f} \mid H^{\perp} \in \hat{A}^{\prime}\right\} \quad$ is a maximal nondetermining subalgebra of $L^{1}(G)$.

We need several elementary lemmas.

Lemma 1.2. $A$ subalgebra $A$ of $L^{1}(G)$ is maximal nondetermining if and only if $A$ is maximal among the proper subalgebras of $L^{1}(G)$ such that $\hat{A}=\hat{A^{-}} \cap L^{1^{\wedge}}(G)$.

Proof. Assume that $A$ is maximal nondetermining. Let $A_{1}$ $=\left\{f \in L^{1}(G): \hat{f} \in \hat{A}^{-}\right\}$. Then $A \subset A_{1}$ and $\hat{A}^{-}=\hat{A}_{1}^{-}$. Since $A_{1}$ is then nondetermining, we have $A=A_{1}$. Hence $\hat{A}=\hat{A}_{1}=\hat{A}^{-} \cap L^{1}(G)$ by definition of $A_{1}$. Furthermore, if $B$ is a subalgebra of $L^{1}(G)$ such that $A \varsubsetneqq B \varsubsetneqq L^{1}(G)$, then $\hat{A} \varsubsetneqq \hat{B}_{\ddagger}^{\subsetneq} L^{1^{\wedge}}(G)$ as Fourier transform is an isomorphism. So $B$ is determining, or $\hat{B}^{-}=L^{1^{\wedge}}(G)^{-}=C_{0}(\Gamma)$. Therefore, $\hat{B} \not \hat{B}^{-} \cap L^{1^{\wedge}}(G)$.

Now let $A$ be a subalgebra of $L^{1}(G)$ being maximal relative to the property that $\hat{A}=\hat{A}^{-} \cap L^{1^{\wedge}}(G)$ and suppose $A \varsubsetneqq B \subset L^{1}(G)$. Let $B_{1}=\left\{f \in L^{1}(G): \hat{f} \in \hat{B}^{-}\right\}$. Then clearly $\hat{A} \subsetneq \hat{B} \subseteq \hat{B}_{1}=\hat{B}^{-} \cap L^{1^{\wedge}}(G) \subseteq \hat{B}^{-}$. So $\hat{B}_{1}^{-}=\hat{B}^{-}$. Thus $\hat{B}_{1}=\hat{B}^{-} \cap L^{1^{\wedge}}(G)=\hat{B}_{1}^{-} \cap L^{1 \wedge}(G)$. But by our choice of $A$, we must have $B_{1}=L^{1}(G)$. Hence $\hat{B}^{-}=\hat{B}_{1}^{-}=C_{0}(\Gamma)$, i.e., $B$ is determining.

A direct consequence of this lemma is that a maximal nondetermining subalgebra is closed in $L^{1}$-norm as Fourier transform is normdecreasing.

Lemma 1.3. Let $A \subset L^{1}(G)$ and let $E \subset \Gamma$ be the hull of a closed ideal I of $L^{1}(G)$ contained in $A$. Then $\hat{I}^{-}=\left\{f \in C_{0}(\Gamma): f \mid E=0\right\} \subset \hat{A}^{-}$.

Proof. First, we show that for every closed ideal $I$ of $L^{1}(G), \hat{I}^{-}$ is a closed ideal of $C_{0}(\Gamma)$. Let $f \in \hat{I}^{-}$and $g \in C_{0}(\Gamma)$. It suffices to show $f \cdot g \in \hat{I}^{-}$. Pick $\left\{f_{n}\right\} \subset I$ and $\left\{g_{n}\right\} \subset L^{1}(G)$ such that $\left\{\hat{f}_{n}\right\}$ and $\left\{\hat{g}_{n}\right\}$ converge to $f$ and $g$ respectively in sup-norm since $L^{1^{\wedge}}(G)$ is dense in $C_{0}(\Gamma)$. Therefore the sequence $\left\{\hat{f}_{n} \cdot \hat{g}_{n}\right\}$, i.e. $\left\{\left(f_{n} * g_{n}\right)^{\wedge}\right\}$, converges to $f \cdot g$. But for each $n, f_{n} * g_{n} \in I$. So $f \cdot g \in \hat{I}^{-}$. 
Let $\hat{I}^{-}=\left\{f \in C_{0}(\Gamma): f \mid F=0\right\}, F=$ hull $_{C_{0}(\Gamma)}\left(\hat{I}^{-}\right)$. It remains to show $F=E$. As $f \in \hat{I}^{-}$implies $f \mid E=0$, so $E \subseteq F$. On the other hand, let $J=\left\{f \in L^{1}(G): \hat{f} \mid F=0\right\} . \hat{J}=\hat{I}^{-} \cap \hat{L}^{1} \supset \hat{I}$ implies $J \supset I$. Thus $F \subseteq E$. Therefore $E=F$.

Corollary 1.4. Let $A \subset L^{1}(G)$ be maximal nondetermining. Then for every closed ideal I of $L^{1}(G)$ contained in $A$, the largest closed ideal of $L^{1}(G)$ having the same hull as $I$ is also contained in $A$.

Leмma 1.5. Let $E$ be a closed subset of $\Gamma$ and $A$ be a closed subalgebra of $L^{1}(G)$ such that $A$ contains the smallest closed ideal of $L^{1}(G)$ having $E$ as hull. Then $\hat{A}^{-} \mid E=(\hat{A} \mid E)^{-}$.

Proof. (i) It is always true that $\hat{A}^{-} \mid E \subseteq(\hat{A} \mid E)^{-}$. For let $f \in \hat{A}^{-} \mid E$, then there exists a sequence $\left\{f_{n}\right\}$ in $A$ and $g$ in $C_{0}(\Gamma)$ such that $\left\{\hat{f}_{n}\right\}$ converges to $g$ and $g \mid E=f$. In particular, $\hat{f}_{n} \mid E$ converges to $g \mid E$, i.e. g|E $=f \in(\hat{A} \mid E)^{-}$.

(ii) To show $(\hat{A} \mid E)-\subset \hat{A}^{-} \mid E$, let $f \in(\hat{A} \mid E)$ - and let $\left\{f_{n}\right\} \subset A$ such that $\left\{\hat{f}_{n} \mid E\right\}$ converges to $f$. Extend $\left\{\hat{f}_{n} \mid E\right\}$ to a Cauchy sequence $\left\{g_{n}\right\}$ in $C_{0}(\Gamma)$. So $\left\{g_{n}\right\}$ converges to certain $g \in C_{0}(\Gamma)$. But $\left(g_{n}-\hat{f}_{n}\right) \mid E$ $=0$ implies that $g_{n}-\hat{f}_{n} \in \hat{A}^{-}$by Lemma 1.3. Hence $\left\{g_{n}\right\} \subset \hat{A}^{-}$and $g \in \hat{A}^{-}$. Therefore $g\left|E=\lim _{n} g_{n}\right| E=\lim _{n} \hat{f}_{n} \mid E=f$, i.e. $f \in \hat{A}^{-} \mid E$.

Proof of the Theorem. (i) Suppose $A$ is reducible. By the usual Zorn's Lemma argument, $A$ contains a largest closed ideal $I$ of $L^{1}(G)$ and the closed subgroup $\Lambda$ generated by $\operatorname{hull}(I)=Z(I)$ is a proper subgroup of $\Gamma$. We claim that $\Lambda$ is the subgroup asserted in the theorem. The minimality is clear. The uniqueness of $\Lambda$ follows from the fact that the largest closed ideal $I$ of $L^{1}(G)$ contained in $A$ is unique. For if $I_{1}$ and $I_{2}$ are two distinctive such, then the closure of the sum of $I_{1}$ and $I_{2}$ is also a closed ideal of $L^{1}(G)$ contained in $A$ properly as $L^{1}(G)$ is regular and $A$ is not an ideal. But $I_{\neq}^{\supset} I_{1}, I_{2}$, which is a contradiction.

Next, we show that $\pi(\hat{A} \mid \Lambda)$ is maximal nondetermining. By Lemma 1.2 it suffices to show that $\hat{A} \mid \Lambda$ is a maximal closed subalgebra of $L^{1^{\wedge}}\left(G / \Lambda^{\perp}\right)$.

Let $f \in(\hat{A} \mid \Lambda)-\cap L^{1^{\wedge}}\left(G / \Lambda^{\perp}\right)$, then $f \in \hat{A}^{-} \mid \Lambda$ by Lemma 1.5. So $f \in\left(\hat{A}^{-} \cap L^{1^{\wedge}}(G)\right)|\Lambda=\hat{A}| \Lambda$ by Lemma 1.2 . Hence $\hat{A} \mid \Lambda$ is closed in $L^{1^{\wedge}}\left(G / \Lambda^{\perp}\right)$. I t is maximal, as any proper closed subalgebra in $L^{1^{\wedge}}\left(G / \Lambda^{\perp}\right)$ containing $\hat{A} \mid \Lambda$ corresponds back in $L^{1^{\wedge}}(G)$ to a proper closed subalgebra containing $\hat{A}$, hence must be $\hat{A}$. That $\pi(\hat{A} \mid \Lambda)$ is irreducible is clear by virtue of our choice of $\Lambda$.

(ii) Now let $A^{\prime}$ be a maximal nondetermining subalgebra of $L^{1}(G / H)$. Let $\Lambda=H^{\perp}$ and $A=\left\{f \in L^{1}(G): \hat{f} \mid \Lambda \in \hat{A}^{\prime}\right\}$. We will show 
that $A$ is maximal nondetermining. Suppose $A \nsubseteq A_{1} \subset L^{1}(G)$, we want to show that $\hat{A}_{1}$ is uniformly dense in $C_{0}(\Gamma)$. Since

$$
A \supset\left\{f \in L^{1}(G): \hat{f} \mid \Lambda=0\right\}
$$

implies that $\hat{A}^{-} \supset\left\{g \in C_{0}(\Gamma): g \mid \Lambda=0\right\}=I$ by Lemma 1.3 , so $\hat{A}_{1}^{-} \supset I$. Hence if $\hat{A}_{1}^{-} \varsubsetneqq C_{0}(\Gamma)$, then $\hat{A}_{1}^{-} \mid \Lambda_{\neq} C_{0}(\Lambda)$. On the other hand, by Lemma $1.5, \hat{A}_{1}^{-} \mid \Lambda=\left(\hat{A}_{1} \mid \Lambda\right)^{-}$. Also $\left(\hat{A}_{1} \mid \Lambda\right)^{-}=C_{0}(\Lambda)$ since $\hat{A}_{1}|\Lambda \not \hat{A}| \Lambda$ $=\hat{A}^{\prime}$ and $A^{\prime}$ is maximal nondetermining. Thus $\hat{A}_{1}^{-} \mid \Lambda=C_{0}(\Lambda)$, a contradiction.

2. In this section, we will discuss the Shilov boundary $S(A)$ and the regular maximal ideal space $M(A)$ of maximal nondetermining subalgebras $A$.

TheOREM 2.1. Let $A$ and $A^{\prime}$ be the corresponding maximal nondetermining subalgebras of $L^{1}(G)$ and $L^{1}(G / H)$ respectively as in Theorem 1.2. Suppose that they are point-separating, then $M(A)=\Gamma$ if and only if $M\left(A^{\prime}\right)=\Lambda=H^{\perp}$.

Proof. (i) Assume $M(A)=\Gamma$. Obviously every $\phi \in M\left(A^{\prime}\right)$ induces a unique $\tilde{\phi} \in M(A)=\Gamma$ via $\delta(f)=\phi \circ \pi(\hat{f} \mid \Lambda)$ ( $\pi$ as in Theorem 1.2). So $\tilde{\phi}(f)=\hat{f}(\gamma)$ for certain $\gamma \in \Gamma$. We claim that $\gamma \in \Lambda$. For $\gamma \in \Lambda$ implies that there exists $f$ in $L^{1}(G)$ such that $\hat{f} \mid \Lambda=0$ and $\hat{f}(\gamma)=1$ by regularity of $L^{1}(G)$. But $\hat{f} \mid \Lambda=0$ implies $f \in A$ and $\pi(\hat{\jmath} \mid \Lambda)=0 \in L^{1}(G / H)$. Now $\tilde{\phi}(f)=\hat{f}(\gamma)=1$ and on the other hand $\tilde{\phi}(f)=\phi \circ \pi(\hat{f} \mid \Lambda)=\phi(0)=0$, a contradiction. Hence $\bar{\phi}$ corresponds to $\gamma \in \Lambda$ and so does $\phi$. The topology of $\Lambda$ is correct by the usual argument. Therefore, $M(A)=\Gamma$ implies that $M\left(A^{\prime}\right)=\Lambda$.

(ii) Suppose now $M\left(A^{\prime}\right)=\Lambda$ and $A=\left\{f \in L^{1}(G) ; \hat{f} \mid \Lambda \in \hat{A}^{\prime}\right\}$. Let $I=\left\{f \in L^{1}(G): \hat{f} \mid \Lambda=0\right\}$. Then $I \subset A$. Consider the mapping $\sigma: A \rightarrow A^{\prime}$ defined by $\sigma(f)=F$ if and only if $\hat{f} \mid \Lambda=\hat{F}$. Clearly, $\operatorname{ker} \sigma=I$. So $A / I$ is isomorphic to $A^{\prime}$. Thus $M\left(A^{\prime}\right)$ is homeomorphic to $M(A / I)$. So $M\left(A^{\prime}\right)=\Lambda$ implies that $M(A / I)=\Lambda$. Regarding $I$ as a closed ideal of $L^{1}(G)$, we have $M(I)=\Gamma \backslash \operatorname{lhull}_{L^{1}(G)}(I)$. On the other hand, regarding $I$ as a closed ideal of $A$, we have $M(I)=M(A) \backslash$ hull $_{A}(I)$. But hull $_{A}(I)=M(A / I)=\Lambda$, so $M(A) \backslash \Lambda=\Gamma \backslash \Lambda$. Since each of the equalities involved so far stands for correspondence which is topologically a homeomorphism and set theoretically the identity map, we have $M(A)=\Gamma$.

The following lemma is direct.

LemMa 2.2. Let $A$ be a closed determining subalgebra of $L^{1}(G)$. Then $S(A)=M(A)=\Gamma$.

A more general form of the lemma is 
LEMMA 2.2.1. Let $B$ be a commutative Banach algebra with (without) identity. If $X$ is a compact (locally compact) Hausdorff space such that $S(B) \subseteq X \subseteq M(B)$ and $(\hat{B} \mid X)^{-}=C(X) \quad\left(C_{0}(X)\right.$ respectively), then $M(B)=S(B)=\mathrm{X}$.

Lemma 2.3. Let $A$ be completely irreducible. Then $(\hat{A} \mid F)^{-}=C_{0}(F)$ for all proper closed subsets $F$ of $\Gamma$.

Proof. Consider the algebra $A_{0}=\left\{f \in L^{1}(G): \hat{f} \mid F \in(\hat{A} \mid F)^{-}\right\}$. As $A_{0}$ contains the closed ideal $I(F)$ of $L^{1}(G)$, so $A \nsubseteq A_{0} \subset L^{1}(G)$ and hence $A_{0}$ is determining. Therefore, $\hat{A}_{0}^{-} \mid F=C_{0}(F)$. But $\hat{A}_{0}^{-} \mid F$ $=\left(\hat{A}_{0} \mid F\right)^{-}$by Lemma 1.5. Also $(\hat{A} \mid F)^{-}=\left(\hat{A}_{0} \mid F\right)^{-}$since $\hat{A}\left|F \subset \hat{A}_{0}\right| F$ $C(\hat{A} \mid F)^{-}$by definition of $A_{0}$. Thus $(\hat{A} \mid F)^{-}=C_{0}(F)$.

THEOREM 2.4. Let $A$ be a maximal nondetermining subalgebra of $L^{1}(G)$ such that $\hat{A}$ separates points on $\Gamma$. Then $S(A) \subseteq \Gamma \subseteq M(A)$, and

(i) if $A$ is completely irreducible, then $S(A)=\Gamma$;

(ii) if $A$ contains nontrivial ideals of $L^{1}(G)$ such that the hull $E$ of the largest closed ideal of $L^{1}(G)$ contained in $A$ is a compact $G_{\delta}$ and $1 \in \hat{A}^{-} \mid E$, then $S(A)=\Gamma$. Hence the equality $S(A)=\Gamma$ always attains if $\Gamma$ is compact and metric, or equivalently, $G$ is countable and discrete.

Proof. That $\Gamma \subseteq M(A)$ is clear for restriction of $\phi \in M\left(L^{1}(G)\right)$ to $A$ is in $M(A)$ as $\hat{A}$ separates points on $\Gamma$. The containment $S(A) \subseteq \Gamma$ follows directly from the familiar formula $\|\hat{f}\|_{\infty}=\lim _{n \rightarrow \infty}\left\|f^{n}\right\|^{1 / n}$.

(i) Suppose that $A$ is completely irreducible. If $S(A) \varsubsetneqq \Gamma$, then $S(A)$ is a proper closed subset of $\Gamma$ as $S(A)$ is closed in $M(A)$. Thus $(\hat{A} \mid S(A))^{-}=C_{0}(S(A))$ by Lemma 2.3. It follows then from Lemma 2.2.1 that $S(A)=\Gamma=M(A)$, a contradiction.

(ii) Now let $A$ be as in (ii) of the theorem. As $S(A) \subseteq \Gamma \subseteq M(A)$, $S(A)=S\left((\hat{A} \mid \Gamma)^{-}\right)$, it suffices to show $S\left(\hat{A}^{-}\right)=\Gamma$.

Firstly, we show that $E \subset S\left(\hat{A}^{-}\right)$. Since $\Gamma$ is normal implies that $E$ is a zero-set, there exists $f \in C_{0}^{R}(\Gamma)$ such that $E=f^{-1}[0]$. Clearly we may assume $f \in C_{0}^{R}(\Gamma)$ and $0 \leqq f \supsetneqq 1$. By assumption and Lemma 1.3, $\left\{g \in C_{0}(\Gamma): g \mid E=0\right\} \subset \hat{A}^{-}$. Thus $f \in \hat{A}^{-}$. Also, since $1 \in \hat{A}^{-} \mid E$ and $E$ is compact, by the fact that $\Gamma$ is normal and Lemma 1.3 again, we can find $f_{1} \in \hat{A}^{-}$such that $0 \leqq f_{1} \leqq 1$ and $f_{1} \mid E=1$. Let $h=f_{1}-f$. Since $h \in \hat{A}^{-}$and peaks on $E, S\left(\hat{A}^{-} \mid E\right) \subset S\left(\hat{A}^{-}\right)$. It remains to show $S\left(\hat{A}^{-} \mid E\right)=E$. As $S\left(\hat{A}^{-} \mid E\right)=K \neq E$ implies that $\hat{A}^{-} \mid K=(\hat{A} \mid K)^{-}$ $=C(K)$, hence by Lemma $2.2 .1 K=E=M\left(\hat{A}^{-} \mid E\right)$, a contradiction. Therefore $S\left(\hat{A}^{-} \mid E\right)=E \subset S\left(\hat{A}^{-}\right)$. Suppose $E \subset S\left(\hat{A}^{-}\right) \varsubsetneqq \Gamma$. As $S\left(\hat{A}^{-}\right)$ is a closed subset of $\Gamma$, by regularity of $L^{1}(G)$ there exists a nonzero function $f$ in $L^{1}(G)$ such that $\hat{f} \mid S\left(\hat{A}^{-}\right)=0$. But $E \subset S\left(\hat{A}^{-}\right)$implies $f \in A$, again a contradiction. 
If $\Gamma$ is compact and metrizable, then clearly hulls of closed ideals of $L^{1}(G)$ are compact $G_{\delta}$ 's. Also every maximal nondetermining subalgebra $A$ of $L^{1}(G)$ contains the identity of $L^{1}(G)$. Thus $1 \in \hat{A}\left|E \subset \hat{A}^{-}\right| E$. Hence the last assertion follows.

3. Applications and examples. A proof of the following theorem can be found in Rudin ([4], Chapter 9).

Theorem 3.1 (Wermer, Simon). Let $G$ be a locally compact abelian group and $S$ a Borel semigroup in $G$. If $L^{1}(S)=\left\{f \in L^{1}(G): \int_{S^{\prime}}|f|=0\right\}$ is a maximal subalgebra of $L^{1}(G)$, then $L^{1}(S)=L^{1}\left(G^{+}\right)$where $G^{+}$is a maximal closed semigroup in $G$ inducing an archimedean order in $G$. Conversely, if $G$ is a locally compact abelian group and $G^{+}$is a semigroup in $G$ inducing an archimedean order in $G$, then $L^{1}\left(G^{+}\right)$is a maximal subalgebra of $L^{1}(G)$.

CoROLlARY 3.2. Let $G$ be discrete and torsion-free and $G^{+}$a maximal closed semigroup in $G$ inducing an order in $G$. Let $\Lambda=G_{0}^{\perp}=\left(G^{+} \cap G^{-}\right)^{\perp}$. Then the algebra $A=\left\{f \in L^{1}(G): \hat{f} \mid \Lambda \in L^{1^{\wedge}}\left(G^{+} / G_{0}\right)\right\}$ is a maximal nondetermining subalgebra of $L^{1}(G)$.

Proof. $G_{0}=G^{+} \cap G^{-}$is clearly a closed subgroup of $G . G^{+} / G_{0}$ is then a closed maximal semigroup in $G / G_{0}$ inducing an archimedean order on $G / G_{0}$. Thus the corollary follows from Theorems 1.1 and 3.1.

CoROLlaRY 3.3. Let $G$ be discrete and have a proper nontrivial torsion subgroup $G_{t}$. Then $L^{1}(G)$ contains maximal nondetermining subalgebras reducible to completely irreducible ones.

Proof. The quotient group $G / G_{t}$ is discrete and torsion-free. So the assertion follows from the above corollary and Theorem 1.1.

As a particular example, take $G=T_{d}$, the circle group with discrete topology. Then $\hat{G}=\bar{Z}$, the Bohr compactification of $Z$. $G$ has nontrivial proper torsion subgroup, so $L^{1}(G)$ always has a maximal nondetermining subalgebra which can be reduced to a completely irreducible one.

CoROllary 3.4. Let $G$ be a disconnected but nondiscrete group. Then $L^{1}(G)$ has maximal nondetermining subalgebras reducible to the type $L^{1}\left(G^{\prime+}\right)$.

Proof. $G$ is disconnected implies that $G$ has nontrivial proper open and closed subgroup $G_{0}$. Hence the quotient group $G / G_{0}$ is discrete. By Corollaries 3.2 or $3.3, L^{1}\left(G / G_{0}\right)$ has maximal nondetermining subalgebras which can be reduced to the form $L^{1}\left(G^{\prime+}\right)$, and so has $L^{1}(G)$ by Theorem 1.1 . 
The following is a discussion due to Rudin [4]. As it does indicate a general way of getting maximal subalgebras of $L^{1}(G)$ from maximal function algebras on certain compact subsets of $\Gamma$, we quote it here as a theorem. We recall first that a compact subset $E$ of a locally compact abelian group $\Gamma$ is called a Helson set if $L^{1^{\wedge}}(G) \mid E=C(E)$, where $G=\hat{\Gamma}$.

TheOREM 3.5 (RUDIN). Let $G$ be a locally compact abelian group which is not compact. Let $E \subset \Gamma$ be a Helson set. If $B \subset C(E)$ is a maximal function algebra on $E$, then $A=\left\{f \in L^{1}(G): \hat{f} \mid E \in B\right\}$ is a maximal subalgebra of $L^{1}(G)$. $A$ is point-separating and nondetermining.

If $G$ is noncompact, then $\Gamma$ is nondiscrete and contains a Cantor set $E$ which is also a Helson set. Rudin [5] showed that $C(E)$ has a maximal subalgebra $B$ which is a function algebra. Thus it follows immediately from the theorem that

COROllaRY 3.6. For every locally compact but noncompact abelian group $G, L^{1}(G)$ contains a maximal point-separating subalgebra which is nondetermining.

In view of Corollaries 3.2 and 3.6, we may naturally raise the question: Does every maximal or maximal nondetermining subalgebra of the group algebra of a discrete group necessarily belong to one of the two categories (up to isomorphism) described in Corollary 3.2 and Corollary 3.6?

The answer is negative. We can easily get an example for $G=Z^{\infty}$ (the direct sum of countably many copies of $Z$ ) by combining a result of Wermer and Browder [6] and Theorem 3.5.

EXAmple 3.7. Let $G=Z^{\infty}$, then $\Gamma=T^{\omega}$ (the complete direct sum of countably many copies of $T$ ). $T^{\omega}$ contains ([4], Chapter 5) an arc $L$, which is a Kronecker set. Explicitly,

$$
L=\left\{x(t)=\left(2 \pi t, 2 \pi t^{2}, 2 \pi t^{3} \cdots\right): 0<\alpha \leqq t \leqq \beta<1, \alpha, \beta, \text { fixed }\right\} .
$$

Being a compact Kronecker set, $L$ is then a Helson set of $\Gamma$.

Next, let $B$ be a maximal subalgebra of $C(J)$, where $J$ is an arc, constructed by Wermer and Browder [6]. Namely, let $J$ be a particular arc of positive Lebesque density at each of its points in the complex plane $\boldsymbol{C}$. Let $\boldsymbol{C}_{\infty}$ be the Riemann sphere. Let $B_{1}=\left\{f \in C\left(\mathbf{C}_{\infty}\right)\right.$ : $f$ is analytic off $J\}$. Then $B_{1} \mid J=B C C(J)$ is a maximal essential algebra on $J$.

$J$ is homeomorphic to $L$. So $C(J)$ is topologically isomorphic to $C(L)$. Thus the image $B_{L}$ of $B$ is a maximal function algebra on $L$. Therefore the algebra $A_{L}=\left\{f \in L^{1}\left(Z^{\infty}\right): \hat{f} \mid L \in B_{L}\right\}$ is maximal in $L^{1}\left(Z^{\infty}\right)$ by Theorem 3.5. 
It remains to show that $A_{L}$ is not isomorphic to any maximal nondetermining subalgebra of $L^{1}\left(Z^{\infty}\right)$ obtained via Corollary 3.2 or 3.6.

Firstly, we observe that $A_{L}$ is not isomorphic to any subalgebra of $L^{1}\left(Z^{\infty}\right)$ of the form $L^{1}\left(Z^{\infty+}\right)\left(Z^{\infty+}\right.$ is a semigroup in $Z^{\infty}$ inducing an archimedean order in $Z^{\infty}$ ) by the following general but simple lemma.

Lemma 3.8. A completely irreducible maximal nondetermining subalgebra $A$ of $L^{1}(G)$ is not isomorphic to any noncompletely irreducible one.

Proof. We show first that $A \subset L^{1}(G)$ is not completely irreducible implies that $A$ is not an integral domain. Let $I \neq\{0\}$ be a closed ideal of $L^{1}(G)$ contained in $A$ and $E=Z(I)$. Pick $f \in L^{1}(G), \hat{f}$ has compact support $F$ disjoint from $E$ and $E \cup F_{\not \neq}^{\complement} \Gamma$. Then pick $g \in L^{1}(G), \hat{g}$ has compact support disjoint from $E \cup F$. So $f, g \in I \subset A$. But $f * g=0$, a contradiction.

Since being an integral domain is a pure algebraic property, the lemma will be completed if we show that a maximal nondetermining subalgebra $A$ which is not an integral domain is not completely irreducible. Let $f, g \in A$ such that $f * g=0$. So $f \mid U=0, U=\operatorname{carrier}(g)$. Since $\Gamma$ is normal, there exists an open subset $V$ of $\Gamma$ such that $\phi \neq V \subset \bar{V} \subset U$. Let $h \in C_{0}(\Gamma)$. By Lemma 2.3, there is a sequence $\left\{f_{n}\right\}$ of $A$ such that $\left\|\hat{f}_{n}-h\right\|_{V^{\prime}} \rightarrow 0$. So $\left\|\left(f * f_{n}\right)^{\wedge}-\hat{f} \cdot h\right\|_{\infty} \rightarrow 0$ on $\Gamma$. Hence $\left(\hat{f} \cdot C_{0}(\Gamma)\right)^{-}=I_{1} \subset \hat{A}^{-}$, and $I_{1}$ is a closed ideal in $C_{0}(\Gamma)$ containing $\hat{f}$. By Lemma 1.2, $\hat{A}=\hat{A}^{-} \cap L^{1^{\wedge}}(G)$. So $A \supset I=\left\{h \in L^{1}(G): \hat{h} \mid Z\left(I_{1}\right)=0\right\}$, which is a closed ideal of $L^{1}(G)$.

Now let $E$ be an independent Cantor set in $T^{\omega}$ which is also a Helson set. Let $B_{E}$ be a maximal essential subalgebra of $C(E)$ and $A_{E}$ $=\left\{f \in L^{1}\left(Z^{\infty}\right): \hat{f} \mid E \in B_{B}\right\}$ be the corresponding maximal subalgebra of $L^{1}\left(Z^{\infty}\right)$. We claim: $A_{L}$ is not isomorphic to $A_{E}$. To show this, we prove the following general assertion:

Lemma 3.9. Let $G$ be discrete. Lot $A_{1}$ and $A_{2}$ be maximal nondetermining, point-separating subalgebras of $L^{1}(G)$ such that the largest closed ideal $I_{i}$ of $L^{1}(G)$ contained in $A_{i}$ has hull $E_{i}, i=1,2$. If further, $\hat{A}_{i}^{-} \mid E_{i}$ is a maximal algebra (it is clearly essential) on $E_{i}$ for each $i_{\text {, }}$ then $A_{1}$ is isomorphic to $A_{2}$ implies that $A_{1} / I_{1}$ is isomorphic to $A_{2} / I_{2}$.

Proof. Let $\sigma$ be an isomorphism from $A_{1}$ onto $A_{2}$. It follows from the facts that each $A_{i}$ is closed and that $\Gamma \subseteq M\left(A_{i}\right)$ that both $A_{1}$ and $A_{2}$ are semisimple Banach algebras. Thus $\sigma$ is open as well as closed.

Consider now the pairs $\left(I_{1}, A_{1}\right)$ and $\left(\sigma\left(I_{1}\right), A_{2}\right)$ and the following diagram: 


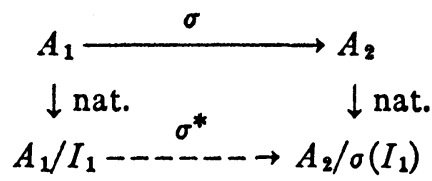

$A_{2} / \sigma\left(I_{1}\right)$ makes sense and is a Banach algebra under quotient norm as $\sigma\left(I_{1}\right)$ is clearly a closed ideal of $A_{2}$.

$\mathrm{By}$ induced mapping theorem, there is an isomorphism $\sigma^{*}$ from $A_{1} / I_{1}$ onto $A_{2} / \sigma\left(I_{1}\right)$ such that the diagram is commutative. As $A_{1} / I_{1} \cong$ iso $\hat{A}_{1}\left|E_{1} \subseteq \hat{A}_{1}^{-}\right| E_{1}$ which by assumption is an integral domain, hence $A_{2} / \sigma\left(I_{1}\right)$ is also an integral domain. We will show that $K=\left\{\gamma \in \Gamma: \hat{f}(\gamma)=0, f \in \sigma\left(I_{1}\right)\right\} \subseteq E_{2}$.

Clearly $K \neq \varnothing$ and contains more than one point. Suppose $K \Phi E_{2}$, then there is a point $p_{1} \in K \backslash E_{2}$. Let $p_{2}$ be another point of $K$. By regularity of $L^{1}(G)$, there exist functions $f$ and $g$ in $L^{1}(G)$ such that $\hat{f}\left(p_{1}\right)=1, \hat{f} \mid U=0, U$ an open set containing $E_{2} \cup\left\{p_{2}\right\}$; and $g|\{\operatorname{supp}(\hat{f}) \cap K\}=0, \hat{g}|\left\{\left\{p_{2}\right\} \cup E_{2}\right\}=1$. So $(f * g) \wedge \mid K=0$ but $\hat{f} \mid K$ $\neq 0, \hat{g} \mid K \neq 0$. Also, by Corollary 1.4 and the fact that $1 \in A_{2}$ that both $f$ and $g$ are in $A_{2}$ as $\hat{f} \mid E_{2}=0$ and $\hat{g} \mid E_{2}=1$. Thus $A_{2} / \sigma\left(I_{1}\right) \cong \hat{A}_{2} \mid K$ and is not an integral domain, a contradiction. Therefore $K \subseteq E_{2}$ and so $I_{2} \subseteq \sigma\left(I_{1}\right)$. Interchanging the roles of $A_{1}$ and $A_{2}$ and considering the inverse map $\sigma^{-1}$ of $\sigma$, we get $I_{1} \subseteq \sigma^{-1}\left(I_{2}\right)$. Consequently $\sigma\left(I_{1}\right)=I_{2}$. So $A_{1} / I_{1}$ is isomorphic to $A_{2} / I_{2}$.

Since $S\left(A_{i} / I_{i}\right)=S\left(\left(\hat{A}_{i} \mid E_{i}\right)^{-}\right)=S\left(\hat{A}_{1}^{-} \mid E_{i}\right)=E_{i}$, and since the adjoint map of the isomorphism from $A_{1} / I_{1}$ onto $A_{2} / I_{2}$ is a homeomorphism from $S\left(A_{2} / I_{2}\right)$ onto $S\left(A_{1} / I_{1}\right)$, we have

Corollary 3.10. Let $G, A_{i}$ and $E_{i}$ be as in the lemma. Then $A_{1}$ is isomorphic to $A_{2}$ implies that $E_{1}$ is homeomorphic to $E_{2}$.

Now if we let $G=Z^{\infty}, A_{1}=A_{L}, A_{2}=A_{E}$ as discussed above. Every condition in the lemma is satisfied. If $A_{L}$ is isomorphic to $A_{E}$, then $A_{L} / I_{L} \cong \hat{A}_{L} \mid L=B_{L}$ is isomorphic to $A_{E} / I_{E} \cong \hat{A}_{E} \mid E=B_{E}$. This implies that $S\left(B_{L}\right)=L$ is homeomorphic to $S\left(B_{E}\right)=E$, a contradiction.

Finally, we apply Lemma 3.9 again to show $A_{L}$ is not isomorphic to any maximal nondetermining subalgebra of $L^{1}\left(Z^{\infty}\right)$ reducible to the form $L^{1}\left(G^{\prime+}\right)$ either. This time we let $G=Z^{\infty}, A_{1}=A_{L}, A_{2}=A$ $=\left\{f \in L^{1}\left(Z^{\infty}\right): \hat{f} \mid \hat{G}^{\prime} \in L^{1}\left(G^{+}\right)\right\} . G^{\prime}$ is a certain quotient group of $Z^{\infty}$ with an archimedean order induced by $G^{\prime+}$, and so $\hat{G}^{\prime}$ is a compact connected subgroup of $T^{\omega}$. Since ${L^{1}}^{\wedge}\left(G^{+}\right)^{-}$is an essential maximal subalgebra on $\hat{G}^{\prime}$, Corollary 3.10 applies. As a compact connected abelian group is never homeomorphic to an arc, $A_{L}$ is not isomorphic to $A$. 
However, the situation on the real line is different. By studying the Helson sets of $R$, we know that

Lemma 3.11. Let $B$ be a maximal function algebra on a Helson set of $R$. Then the essential set $E$ of $B$, which is also a Helson set, is a Cantor set.

Proof. (i) Every Helson set $P$ on $R$ is totally disconnected. For otherwise $P \supset I$, a closed interval. Being a closed subset of Helson set, $I$ is also a Helson set. But clearly, $L^{1^{\wedge}}(R) \mid I_{\neq}^{\subsetneq} C(I)$.

(ii) The essential set $E$ of $B$ must be perfect. Suppose $E$ has an isolated point $p$. Since $B \mid E=(B \mid E)^{-}$being essential and maximal on $E$ is quasi-analytic, $f \in B$ and $f \neq 0$ implies $f(p) \neq 0$, which is impossible.

Actually for all compact Hausdorff spaces $X, A$ is a maximal subalgebra of $C(X)$ implies the essential set of $A$ is perfect.

(iii) Thus $E \subset P \subset R$ being perfect, metric and totally disconnected is a Cantor set on $R$. As a closed subset of a Helson set, $E$ itself is also a Helson set.

\section{REFERENCES}

1. H. S. Bear, Complex function algebras, Trans. Amer. Math. Soc. 90 (1959), 383-393.

2. P. Civin and B. Yood, Regular Banach algebras with a countable space of maximal ideals, Proc. Amer. Math. Soc. 7 (1956), 1005-1010.

3. K. Hoffman, and I. M. Singer, Maximal subalgebras of continuous functions, Acta. Math. 103 (1960), 217-241.

4. W. Rudin, Fourier analysis on groups, Interscience, New York, 1962.

5. - Subalgebras of space of continuous functions, Proc. Amer. Math. Soc. 7 (1956), 825-830.

6. J. Wermer and A. Browder, Some algebras of functions on an arc, J. Math. Mech. 12 (1963), 119-130.

TUlane University 